\title{
Design of News Release System
}

\author{
Lei Xiong \\ College of Information Engineering, Jiangxi University of Technology, Jiangxi Nanchang
}

Keywords: News Release; Online Survey; Information System; News Category

\begin{abstract}
Government news release system is able to break the boundaries between government and society or among governments through information and network technology application as well as information resource integration so as to help the public to quickly acquire government information and services via governmental websites. This paper firstly analyzes the news release system and then carries out systematical analysis to make design for the system. Finally, this paper conducts a summary of the overall design and retailed design. The system designed in this paper possesses the following functions: news browsing, classified searching, user message and online survey etc.
\end{abstract}

\section{Overview}

News release system is a government news management system based on news release and content management. Besides, the news release system is operated based on the $\mathrm{B} / \mathrm{S}$ system, which is able to organize disorderly information (including character, picture, and video) to be presented in an orderly sequence. Modern society is an information-oriented society and it is an important part for us to know government news. As a part of information, it has large quantities of information and various categories and the news release system is then put forward. Judging from the perspective of the public, this paper mainly focuses on information and service facing the public while the news release in internal governmental institutions is not directly related to the public. Besides, the function and content of e-government are demonstrated through governmental websites and the external world begins to have a good knowledge of the information of government via governmental websites, therefore an excellent government news release system's establishment is the basis to achieve good performance of e-commerce.

\section{Demand Analysis}

Traditional news release is completed by papers, TV, radio etc which takes a large amount of labor and cost. Yet, its operation process is not transparent enough and the information feedback ability is poor. The emergence of news release aims to improve the imperfect job, which will bring about a new future for government news release.

News release system integrates news release, message administration and online survey which greatly improves the news release efficiency, saving resources and better combine the superior resources so as to make it convenient for users to review a dazzling array of news at home. In addition, the news release system meets three requirements in terms of news browsing, message and online survey: 
News browsing requirement. News browsing requirement refers to the needs of users on government news browsing and news managers have to update information so as to help users conveniently, quickly and accurately review the news. Backstage management is complicated, including timely updating, user management and system condition maintenance. Users don't have to login in to review the news.

Messages requirement. Message is carried out to give opinions for certain news, and users have to register or login in so as to leave a message.

Online survey require ment. Users participate in the online survey so as to respond to the manager of certain problems. Background managers put forward some questions concerning hotspots and timely update based on the responses and users don't have to login in.

Based on the above analysis, this paper designs different operation authorization and login method for background managers and users: news information is open for all users; users can register to leave a message; carry out operation and add online survey based on the database; timely add, delete or modify the information; maintain manager information; maintain user information; deal with data integrity.

\section{Systematical analysis}

Systematical structure. News releasing system is made up of foreground user subsystem and background management system, and the organizational chart is shown in Fig. 1

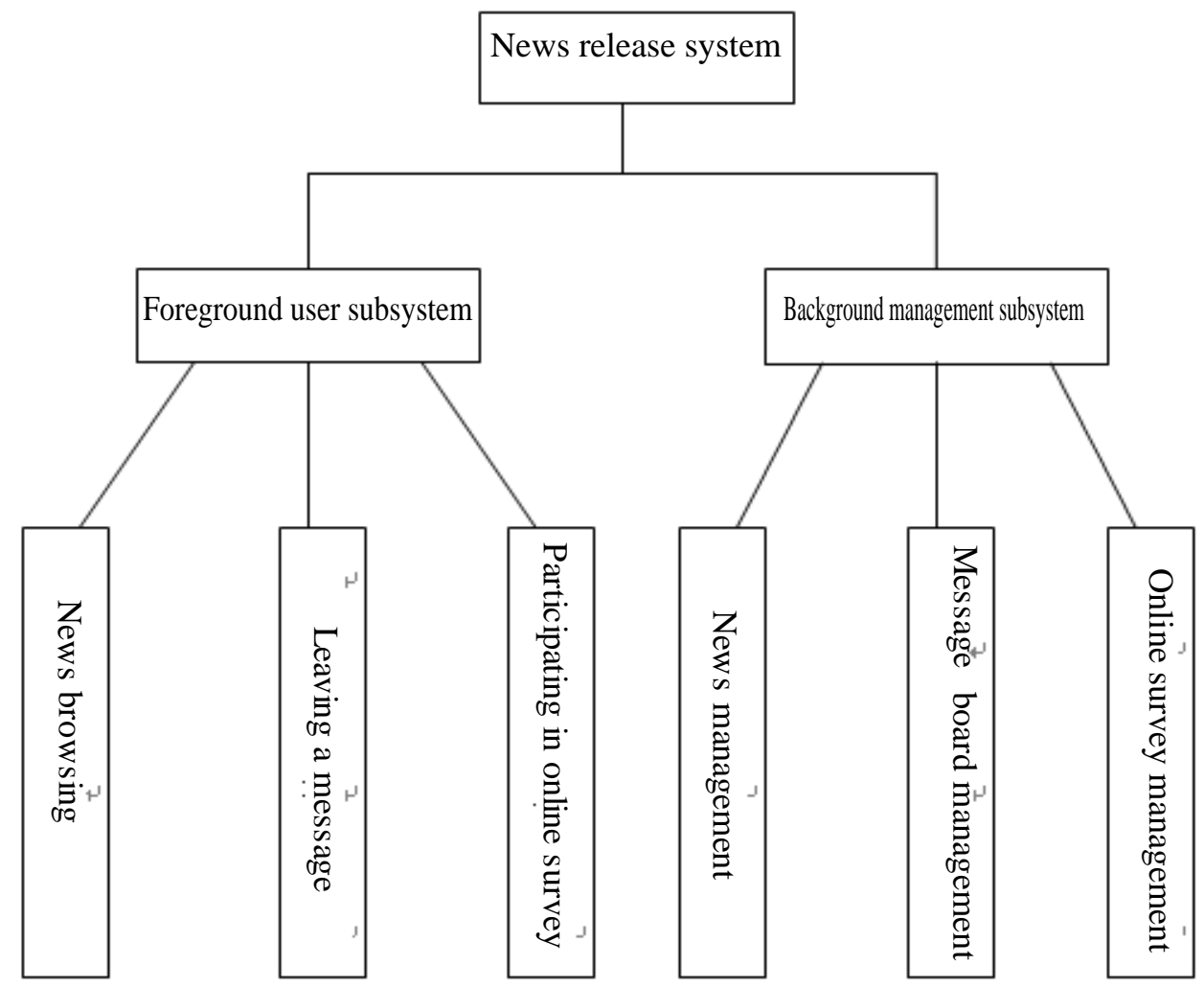

Fig. 1 System organizational chart 
News releasing system includes the operation on foreground user subsystem and background management subsystem: News browsing, classification query, user message, online survey which belong to foreground management. News browsing refers to the condition that users can randomly review the news released by this system; classification query means that users can search for the news they are interested in based on their requirement; message can help users to come up with their own ideas and users have to register an account; online survey help users to respond to the questions proposed by this system; background administrator subsystem can help users to add, modify and delete news; the message management assist managers to properly deal with messages; online survey refers to the condition that mangers put forward questions for users to respond.

Systematical business process analysis. Business process analysis is refined based on the business functions, and it adopts survey information so as to integrate every step into a complete graphic to form a business process diagram. Press release subsystem management manifests itself in two ways: users review news in the foreground, and administrators manage in the background.

As shown in Fig. 2, the process of background subsystem is: the manger log in the admin interface with authorized pass word and information can be managed, added, deleted. In other management, managers can manage the message and online survey carried out by users.

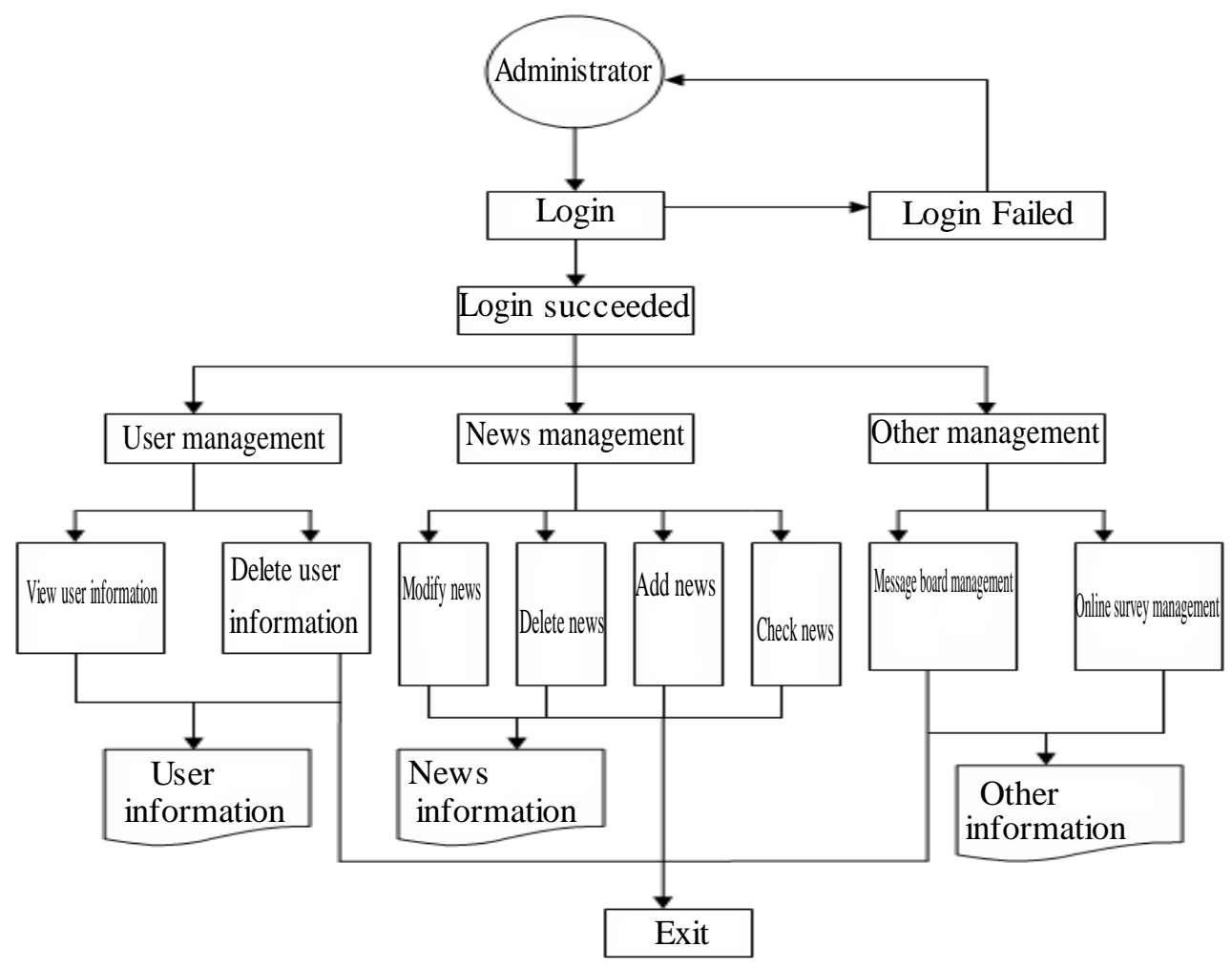

Fig. 2 Flow Chart of system background operation 
As is shown in Fig. 3, the foreground process is that users don't need to log in, and the background information chart can be reviewed and checked in the foreground. Users can choose the news they are interested in according to their own preferences or carry out online survey through registering and logging in.

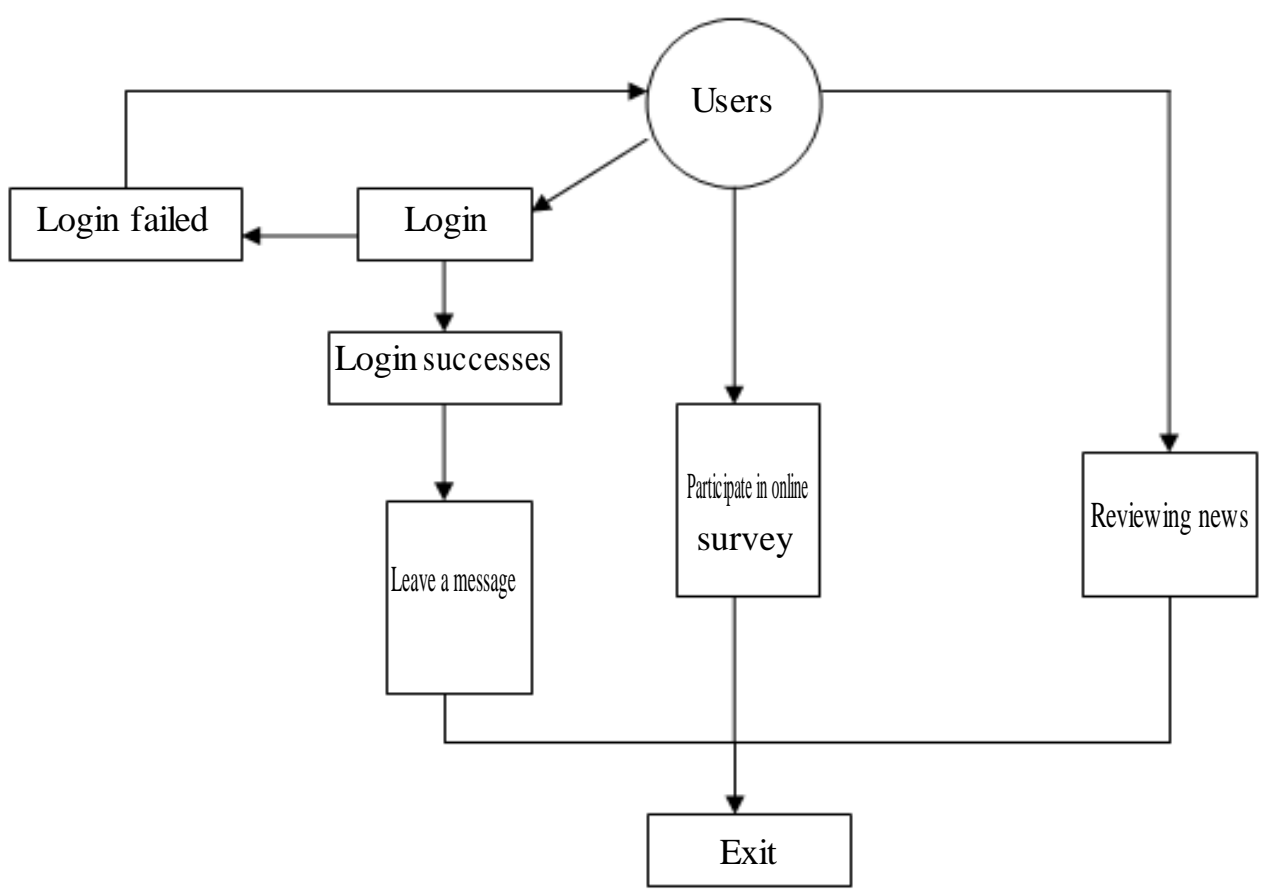

Fig. 3 Flow chart for system foreground

Data management ability request. Able to handle a large volume of news data;

High safe index, prevent hacking;

Strong load capacity to prevent the bad influencing on speed because of excessive data;

Obey the rules of data integrity to guarantee the validity of data;

Guarantee the storage of database engine.

Mirror Snapshot to timely carry out remedy if there should be data loss.

Data recovery: if there should be data damage, it can reduce the loss.

Password management: In order to guarantee the safety of system data, the system should manage the login password.

Requirements for performance. The operation is convenient and flexible

The system is stable;

The system is safe;

The system should possess fault tolerance;

In terms of speed, the foreground can quickly respond to users and there should not be timeout in the background operation.

News release system is developed for releasing government news, and traditional news releasing system is conducted to solve the problem of dynamic release. Yet, the distribution and display is simple with simple functions and different website constructions should be 
satisfied with different systems. News release system is constructed for the background management, which provides managers with a database window, aiming to help those managers without professional computer knowledge to carry out implementation in data so as to complete the data adding, deleting and modifying. At the same time, it can provide foreground users with a friendly and convenient environment.

\section{System Total Design}

System design principle. Compared with common applications, network application focuses more on the stability and robustness while e-commerce asks strict demand on the safety of application. So the main design principle of this system is:

Safety

Its scope includes all aspects from system safety to customer's sensitive data. In addition, measures can be adopted include: hardware and software firewalls, anti-virus measures, anti-hacking measures, different landing authorization mechanism, and so on.

Robustness

It refers to the fault tolerance ability. The news release system adopts high-performance hardware and related software products to ensure customers can always get quality service. Besides, the back-office data is backed up every day, so the site has good recovery ability.

User-friendliness

The users of news release system are members and administers and the interface development still adheres to the principle of customer-orientation, trying the utmost to construct a serious, generous system with multiple functions and is easy to operate to give the excellent support. System development starts from the perspective of members and administrators so as to analyze the possible requirement, aiming to carry out simple and easy operation.

System functions and modules. The news management system should possess following functions

(1) News management

Add, modify and delete news.

(2) Message board management

Manage all of the messages.

(3) Online survey management

Timely add hotspots for users to answer.

(4) System verification

The system login should be verified before carrying out management. It is not necessary for users to login to review the news while system managers have to be authenticated in order to manage the system.

Based on the above function requirement, the system includes the following modules:

(1) News scan homepage

(2) News scan final page

(3) System management homepage

(4) Message module

(5) Online survey module

(6) News release nodule 
(7) News modification module

(7) News deleting module

The system functional module is shown in Fig. 4

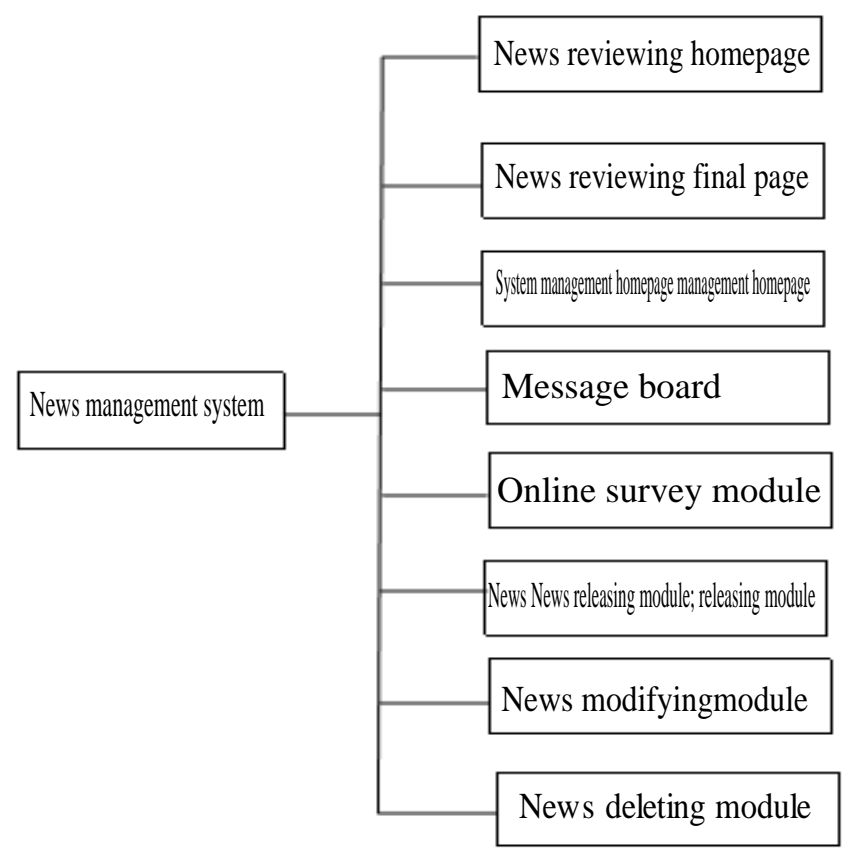

Fig. 4 System functional module

System implementation schemes. B/S (Browser/Server) mode is also known as B/S structure. It emerges along with the Internet technology so as to develop the C/S module. In this structure, the user interface is implemented by IE browser. The most advantageous point of $\mathrm{B} / \mathrm{S}$ module is that its maintenance is simple which can grant different people in different locations with the access to review and operate the data through different approaches (such as LAN, WAN, Internet/Intranet etc).

$\mathrm{B} / \mathrm{S}$ Web structure model is emerged and developed based on the requirement for Intranet. Intranet is a dedicated network within enterprises based on TCP/IP protocol, and it adopts Internet technology and standards such as TCP/IP, HTTP, SMTP, HTML so as to help carry out the internal exchange. Meanwhile, it has the function of connecting Internet and preventing outside intrusion. On the other hand, database has powerful data storage and management capabilities, which can dynamically input and output the data. If the database is applied to Intranet, it can not only realize the online releasing but also provide users with dynamic information retrieval and data processing services.

System database design. The establishment of database is to set up a database and applied technology, which can be regarded as the core technology in the course of information system development and construction. To be specific, database design is aimed at a specific applied environment so as to construct the optimal database model, database and applied system so as 
to efficiently store data, meet the needs of various user applications (information requirements and processing demands).

(1) The setting of data file structure should meet the requirement of management

While designing the data file structure, the management requirement as well as actual processing should be taken into consideration so as to organize the data to simplify the follow-up process of dealing with actual problems.

(2) The data record should embody the management requirement of all levels

Data storage is conducted for management, and if the recorded content cannot meet the requirement of management then the document can be regarded as incomplete. Therefore, in terms of designing the database structure, various information and contents in terms of one aspect should be organized so as to fully and objectively reflect matters and embody a concentrated reflection of each side.

(3) Reduce data redundancy

Data redundancy is one of the indicators to measure the data, which is contradictory to reflect all of the sides of matters because judging from the integrity, the data collections reflecting all of the sides may have cross-tabs and the data redundancy will inevitably occur. So, we can to consider the optimal solutions or satisfactory solution.

(4) Keep data independence

When data is being added, modified or deleted, the data structure as well as relationships should keep the same and the applied process should not depend on the organization method and storage location. That is to say, different applied procedures can access the data in the database according to required data structure. If the organization method is changed, it is necessary to re-prepare or modify the existing procedure.

(5) Keep the integrity and safety of data

While systematically access to data in the database, we have to adopt access permission, authentication, and data stream encryption so as to prevent illegal access and damage. What's more, the data integrity can be protected based on various prevention methods.

(6) Maintain the expanding and updating ability

It is not realistic to permanently use the existing database because things are always changing. So the existing database systems cannot always meet the current needs of users. Besides, there should be corresponding solutions for expanding and updating the system for long-term needs.

\section{Detailed Design of System}

Realization of system interface. User interface exchange and transfer information between human beings and computer, and a user friendly interface directly affects the system's performance and life. Government news website, as a political platform can be beckoned as a window for dispersing information to readers. Therefore, the theme style should be sedate, elegant and serious with blue as the main tune. Besides, soft background color can be matched so as to give readers fresh feelings.

Realization of login user interface

Login user interface is the homepage for a news release system and users can review the political, domestic, international and social news or leave a message and carry out online survey. It has user login and registration selections for users to leave messages. 
Realization of login manager interface

Administrators have their own login access, and they can log in to select the operation order.

Realization of user registration and manager login

In this system, users and administrators are in foreground and background interfaces, so users and administrators respectively possess different landing pages and landing rights. Registered users need to fill in the user name, user password, name, phone number and address.

Through homepage links, the system sets different login pages for managers and users. The login authentication implementation is primarily aiming to establish different charts for them and insert the password into the chart when they register; administrators have dedicated administrator login passwords. When a login authentication is conducted, the landing page is returned to the chart to check the information and if the information is correct, users can login and the information is incorrect, the system will prompt users to return to the error page to login again. Besides, the administrator login is also conducted based on the comparison between the login information and the authority list and if it is same, the page shall go to the operation page and if is different, it will go to the error page.

Realization of news management. News management is realized by adding news, modifying news and deleting news. Adding news is to add information in the system, including political, domestic, international and social news. Modifying news is to modify the information while deleting news aims to delete unnecessary information.

Adding news

Contents have to be filled in include article title, article categories, authors, news content, whether the contents are passed or recommended.

Modifying news

News modifying aims to modify the article title, publication date, and the author. Clicking the modification button, the contents can be modified.

Deleting news

News deleting aims to delete the article title, publication date, and the author. Clicking the deleting button and the contents can be deleted.

Realization of message board. Message board is the one can be entered into after registration and clicking the viewing message button, users can review the recent messages and clicking the button of expecting to leave the message, users can leave a message.

\section{Conclusions}

The news releasing system should be simple, generous and serious so as to provide users with convenient and quick service. The development of news release system should pay attention to the background database design and this system has limitations in terms of function, which is not complete and remains to be improved. As a basis for information release system, good database design system can help to strengthen the system and better use the information. Besides, the database connection is also an important sector of this design. Firstly, we have to draw the flow chart and then design the information chart and we have to take into consideration of comprehensive information so as to avoid unnecessary troubles. The news release system which is completed in the end can help to realize the news browsing, message, 
online survey etc. Moreover, the online survey can help to count the percentage so as to directly elaborate problems.

\section{Acknowledgements}

This work was financially supported by project of Technology Department of Jiangxi Province [No 20143BBM26048] and project of Jiangxi University of Technology [No. xtcx201312].

\section{References}

[1] MS SELINA CHA. Development and Application of Electronic System Navigation. First Edition. Beijing: Posts \& Telecom Press, 2004: P1-3.

[2] Yu Xiangdong. Brief Analysis on the E-government Development At Home and Abroad. Information Technology \& Standardization, 2003. Third edition: P14-15

[3] Wang Xiaoyi, Liu Zhongbing, Xu Jiajing etc. Excellent Examples of JSP Database Development. The first edition. Beijing: Publishing House of Electronics Industry, 2005: P9-10

[4] Robert J Brunner, Frank Cohen.Java web services unleashed[M].2002: P32-34

[5] Sa Shixuan, Wang Shan. An Introduction to Database System [M]. Third Edition. Beijing: Higher Education Press, 2002: P4-5

[6] Zhang Haifan. Software Engineering[M]. The first edition. Beijing: Posts \& Telecom Press, 2002:P91-92. 